\title{
Comparison of Cardiac Repolarization After Transcatheter Aortic Valve Implantation and Surgical Aortic Valve Replacement: A Longitudinal Study
}

\author{
Satoru Chino - Eriko Yamanaka - Toshifumi Takasusuki • \\ Shinsuke Hamaguchi · Shigeki Yamaguchi
}

Received: August 29, 2019 / Published online: November 6, 2019

(C) The Author(s) 2019

\begin{abstract}
Introduction: Transcatheter aortic valve implantation (TAVI) has been established as an alternative to surgical aortic valve replacement (SAVR) for high-risk patients. To assess the impact of TAVI on cardiac repolarization, we compared QT dispersion (QTD) and the interval from the peak to the end of the $\mathrm{T}$ wave (Tpeak-Tend: TpTe) between the patients who underwent TAVI and those who underwent SAVR and TpTe between the patients who underwent TAVI or SAVR.

Methods: This retrospective study was approved by the ethics committee of Dokkyo Medical University Hospital. The study included 45 patients who underwent TAVI and 45 patients who underwent SAVR. The QT, corrected QT (QTc), QTD, QTc dispersion $(\mathrm{QTcD})$,
\end{abstract}

Enhanced Digital Features To view enhanced digital features for this article go to https://doi.org/10.6084/ m9.figshare.10059275.

S. Chino - E. Yamanaka · T. Takasusuki (凹) •

S. Hamaguchi · S. Yamaguchi

Department of Anesthesiology, School of Medicine,

Dokkyo Medical University, Kitakobayashi 880,

Mibu, Tochigi 321-0293, Japan

e-mail: takasusu@dokkyomed.ac.jp
Tp-Te, Tp-Te/QT, and Tp-Te/QTc were manually measured in standard 12-lead electrocardiogram (ECG) recordings obtained before surgery, immediately after surgery, 1 month, 3 months, and 6 months after surgery and compared between the two groups.

Results: No change was observed in RR, QT, QTc, Tp-Te, Tp-Te/QT, and Tp-Te/QTc in the two groups throughout the study. The QTD and QTcD significant decreased immediately after surgery in the TAVI group as compared to the SAVR group $(P<0.001)$. In contrast, QTD and QTcD in the SAVR group gradually, but not significantly declined 6 months after surgery.

Conclusions: QTD and QTcD immediately decreased after TAVI as compared to SAVR. Our findings indicate that TAVI more rapidly improved dispersion of spatial repolarization than SAVR.

Keywords: Cardiac repolarization; QT dispersion; Surgical aortic valve replacement; TpeakTend; Transcatheter aortic valve implantation 


\section{Key Summary Points}

Why carry out this study?

To assess the impact of transcatheter aortic valve implantation (TAVI) on cardiac repolarization, we compared QT dispersion (QTD) and Tpeak-Tend (TpTe) between patients who underwent TAVI and those who underwent surgical aortic valve replacement (SAVR).

Our hypothesis is that TAVI might not increase the QTD or TpTe compared to SAVR.

What was learned from the study?

QT dispersion and corrected QT dispersion significantly decreased immediately after surgery in the TAVI group compared with the SAVR group.

Our findings indicate that TAVI more rapidly improved dispersion of spatial repolarization than SAVR.

\section{INTRODUCTION}

In recent times, transcatheter aortic valve implantation (TAVI) has become a routine procedure for high-risk patients who cannot undergo open-heart surgery. However, there have been several reports of associated complications such as vascular injuries, bleeding, stroke, and acute kidney injury (AKI) after TAVI [1]. Moreover, the long-term outcomes of TAVI in the elderly are still uncertain.

Dispersion of the QT interval (QTD), which is defined as the difference between the maximal and minimal QT interval on a 12-lead surface electrocardiogram (ECG), reflects the regional heterogeneity of ventricular repolarization [2] and is considered as an index of lethal ventricular arrhythmia [3]. The increase of QTD might be associated with the risk of cardiac arrest or perioperative cardiac death in patients with aortic stenosis (AS) [4]. Previous study has revealed that increased QTD due to AS immediately improved after TAVI [5].

The prolongation of the interval from the peak to the end of the $\mathrm{T}$ wave (Tpeak-Tend: TpTe) on ECG and TpTe/QT ratio, which reflects the transmural dispersion of ventricular repolarization (TDR), has also been associated with the incidence of ventricular arrhythmia $[6,7]$. Similar to QTD, TpTe is prolonged in patients with severe AS and immediately recovers after TAVI [8].

To compare the impact of surgical aortic valve replacement (SAVR) and TAVI on cardiac repolarization, we measured QTD and TpTe before surgery, immediately after surgery, and 1 month, 3 months, and 6 months after surgery.

\section{METHODS}

Forty-five patients aged 69-95 years who underwent TAVI and 45 patients aged 55-85 years who underwent SAVR at Dokkyo Medical University Hospital between April 2015 and March 2019 were included in the retrospective study. Patients with complete or incomplete bundle branch block, pacemaker rhythm, non-interpretable ECG data, antiarrhythmic drugs with a possible risk of QT prolongation, and significant coronary artery disease diagnosed by coronary angiography (CAG) were excluded from this study. Upon screening the medical records, four patients from the TAVI group and four patients from the SAVR group were excluded due to an indistinct $T$ wave in 12-lead ECGs. All procedures performed in studies involving human participants were in accordance with the ethical standards of the institutional and national research committee and the 1964 Helsinki Declaration and its later amendments or comparable ethical standards. Informed consent was obtained from all the participants and this study was approved by the ethics committee of Dokkyo Medical University (registration number: R-9-13). The study was registered at the UMIN Clinical Trials Registry (registration number: UMIN000033701). 


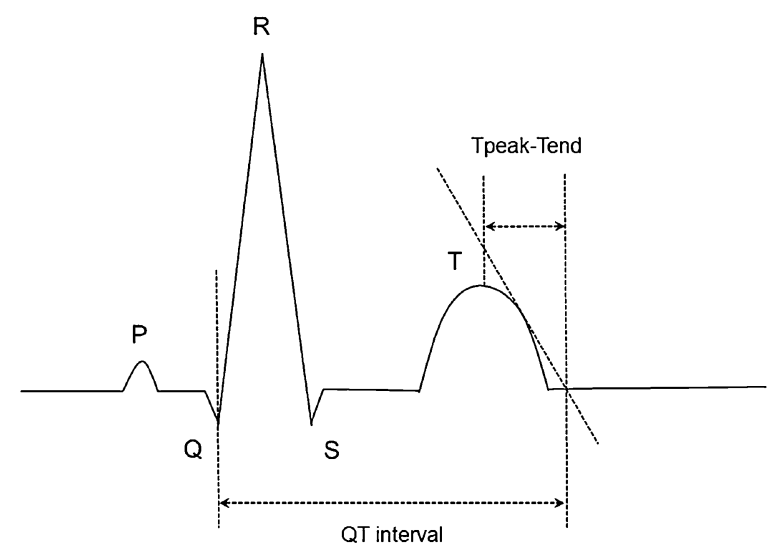

Fig. 1 Measurement of QT interval and Tpeak-Tend using the tangent method

\section{Measurement of QT, QTD and TpTe}

Standard 12-lead ECG recordings were obtained from patients at a paper speed of $25 \mathrm{~mm} / \mathrm{s}$. The RR interval, QT interval, corrected QT (QTc) interval, QTD, QTcD, TpTe interval, TpTe/QT ratio, and TpTe/QTc ratio were manually measured in the ECG recordings. The QT interval in leads II and V5 was measured using the tangent method, and it was considered as the interval from the onset of the $Q$ wave, which was defined as the intersection of a threshold level with the differential of the Q wave, to the end of the $T$ wave, which was defined as the intersection of a tangent to the steepest slope of the last limb of the T wave and the baseline (Fig. 1). The QTc interval was calculated using Fridericia's formula $(\mathrm{QTc}=\mathrm{QT} / 3 \sqrt{ } \mathrm{RR})$. The TpTe interval was measured as the interval from the peak of the $\mathrm{T}$ wave to the end of the $\mathrm{T}$ wave in lead II. The end of the $\mathrm{T}$ wave was determined using the tangent method. Three consecutive cycles in lead II were measured and averaged for analysis of the RR interval, QT interval, and TpTe interval. The QTD was defined as the difference between the maximum and minimum mean QT interval on 12-lead ECG. The mean value of data derived from three successive beats for each lead was used for analysis. Leads in which the end of the $\mathrm{T}$ wave could not be clearly detected were excluded from the study. The investigator who measured these values was blinded to group allocation (TAVI or SAVR group). The ECG recordings were performed before the surgery, immediately after surgery, and 1 month, 3 months, and 6 months after surgery in both groups.

\section{Statistical Analyses}

Data are expressed as mean \pm standard deviation. Patient characteristics were analyzed using the Mann-Whitney $U$ test, Fisher's exact test, and Chi-square test as appropriate. The RR interval, QT interval, QTc interval, QTD, QTcD, TpTe interval, TpTe/QT ratio, and TpTe/QTc ratio were analyzed using the Mann-Whitney $U$ test and analysis of co-variance (ANCOVA) after adjustment of age, sex, and duration of hospitalization. These statistical analyses were performed using IBM SPSS 24.0 (IBM Japan, Tokyo). In all analyses, the probability to detect a significant difference was set at 5\% $(P<0.05)$.

\section{RESULTS}

Table 1 shows the patient characteristics for the present study. There were no significant differences in body mass index (BMI), aortic valve area (AVA), peak gradient, mean gradient, left ventricular ejection fraction (LVEF), and duration to discharge after surgery between the two groups. The number of females was significantly higher in the TAVI group than in the SAVR group. The mean age of the participants in the TAVI group was higher than in the SAVR group $(P<0.0001)$. The height and weight of the participants were significantly higher in the SAVR group as compared to the TAVI group $(P<0.0001)$.

Table 2 shows the evaluation of the RR interval, QT interval (in leads II and V5), QTc interval (in leads II and V5), QT dispersion, QTc dispersion, TpTe, TpTe/QT ratio, and TpTe/QTc ratio in the two groups at each time. Before surgery, no significant difference was observed in RR interval, QT interval, QTc interval, QT dispersion, QTc dispersion, TpTe, TpTe/QT ratio, and TpTe/ QTc ratio between the two groups. The RR, QT, and QTc in leads II and V5 did not change throughout the observation in both groups. The QTD and QTcD were significantly lower 
Table 1 Patient characteristics

\begin{tabular}{|c|c|c|c|}
\hline & TAVI $(n=45)$ & $\operatorname{SAVR}(n=45)$ & $P$ value \\
\hline Sex (male/female) & $9 / 36$ & $22 / 23$ & 0.0073 \\
\hline Age (years) & $84.9 \pm 5.0$ & $74.4 \pm 7.4$ & $<0.0001$ \\
\hline Height $(\mathrm{cm})$ & $145.8 \pm 9.7$ & $155.8 \pm 8.8$ & $<0.0001$ \\
\hline Weight (kg) & $46.0 \pm 10.4$ & $55.1 \pm 11.3$ & $<0.0001$ \\
\hline $\operatorname{BMI}\left(\mathrm{kg} / \mathrm{m}^{2}\right)$ & $21.5 \pm 3.8$ & $22.6 \pm 4.0$ & 0.16 \\
\hline $\operatorname{AVA}\left(\mathrm{cm}^{2}\right)$ & $0.67 \pm 0.17$ & $0.65 \pm 0.22$ & 0.58 \\
\hline Peak gradient $(\mathrm{mmHg})$ & $88.1 \pm 27.2$ & $96.9 \pm 30.4$ & 0.15 \\
\hline Mean gradient (mmHg) & $54.2 \pm 18.7$ & $58.7 \pm 19.2$ & 0.25 \\
\hline LVEF (\%) & $62.1 \pm 10.5$ & $60.0 \pm 13.6$ & 0.42 \\
\hline Duration to discharge after surgery & $21.1 \pm 23.4$ & $24.4 \pm 15.1$ & 0.43 \\
\hline \multicolumn{4}{|l|}{ NYHA classification (\%) } \\
\hline I & 6.7 & 8.9 & 0.706 \\
\hline II & 53.3 & 55.6 & \\
\hline III & 33.3 & 33.3 & \\
\hline IV & 6.7 & 2.2 & \\
\hline Hypertension (\%) & 84 & 73 & 0.18 \\
\hline Diabetes mellitus (\%) & 22 & 33 & 0.21 \\
\hline Hyperlipidemia (\%) & 44 & 47 & 0.76 \\
\hline Smoker (\%) & 20 & 18 & 0.83 \\
\hline Peripheral arterial disease (\%) & 9 & 9 & 0.97 \\
\hline Coronary artery disease (\%) & 29 & 22 & 0.63 \\
\hline
\end{tabular}

Data are presented as mean \pm standard deviation

$B M I$ body mass index, $A V A$ aortic valve area, $L V E F$ left ventricular ejection fraction, $N Y H A$ New York Heart Association

immediately after surgery in the TAVI group than in the SAVR group $(P<0.001)$. The QTD and QTcD in SAVR decreased gradually, but not significantly 6 months after surgery. The TpTe, TpTe/QT, and TpTe/QTc did not change throughout the observation.

Table 3 shows the analyses of ECG variables using a general linear model with analysis of covariance after adjustment of age, sex, and duration to discharge after surgery. After adjustment of age, sex, and duration to discharge after surgery, there were significant differences in QTD and QTcD between TAVI and SAVR immediately after surgery $(P<0.001)$.
There was a significant difference in QT in lead V5 between TAVI and SAVR at 3 months $(P=0.028)$. However, RR, QTc, TpTe, TpTe/QT, and TpTe/QTc were not changed throughout the observation.

\section{DISCUSSION}

\section{Comparison of QT Interval in TAVI and SAVR}

No significant differences in QT and QTc between TAVI and SAVR were observed in the 







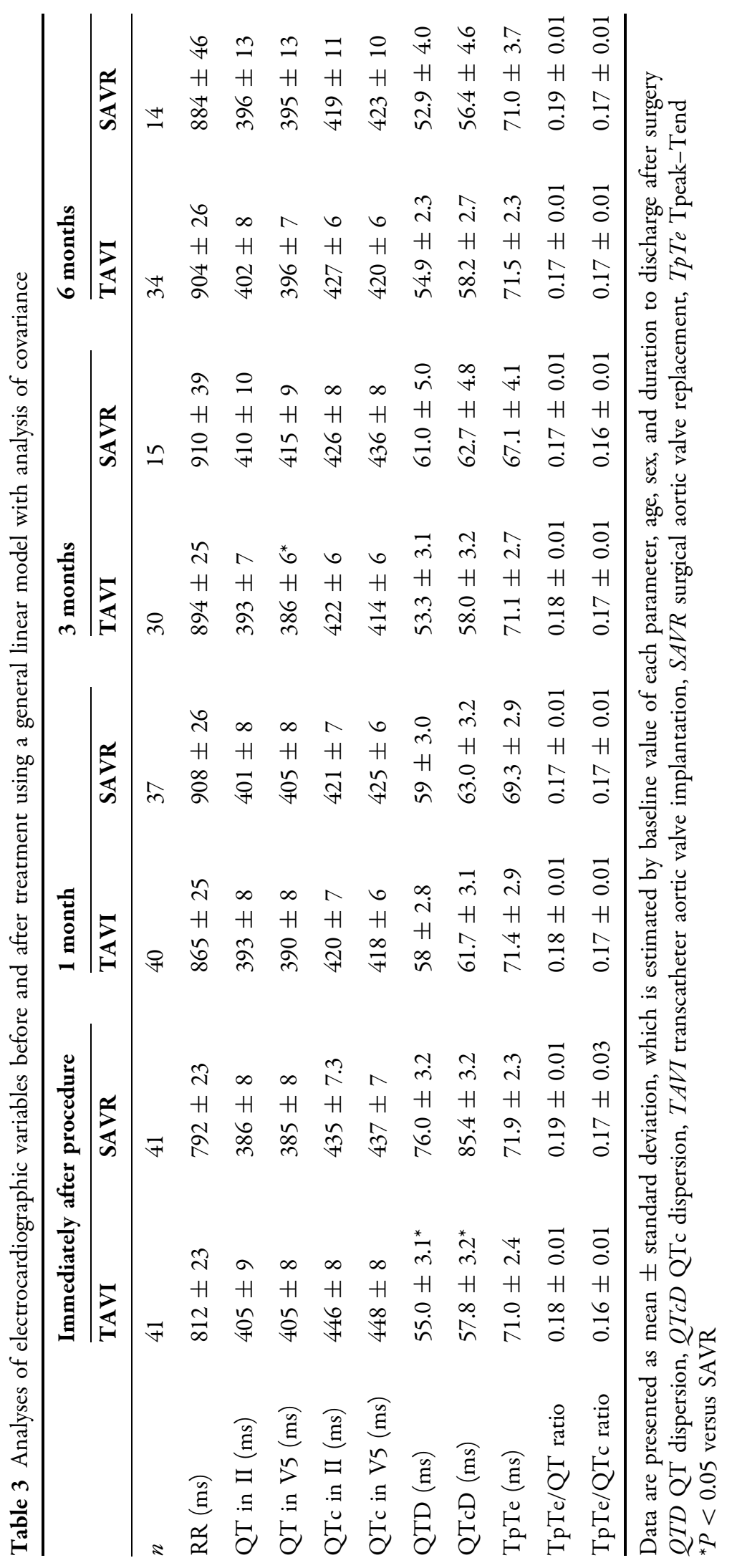


present study. However, QTc, but not QT, was slightly increased immediately after surgery as compared to pre-surgery in both the groups. We also observed decreased RR interval immediately after surgery in both the groups. However, these RR changes recovered on postoperative day 1. The QTc may be overcorrected due to tachycardia after the surgery. Sinus tachycardia, which may be caused by release of catecholamine in response to surgical stress or anemia, is common and subsides within a few days after the surgery. A postoperative tachycardia should not be dismissed within the first 4 days post-surgery [9].

\section{Comparison of QTD Between TAVI and SAVR}

In the present study, QTD and QTcD remained high immediately after surgery in the SAVR group, whereas QTD and QTcD in the TAVI group decreased. These differences in QTD and QTcD eliminated 1 month after surgery. QTD is increased in most patients with AS and these changes are weakly related to the degree of left ventricular hypertrophy [4]. Several studies have shown that increased QTD due to AS, which is associated with the risk of ventricular arrhythmia, decreases after TAVI [7, 10]. Our findings also confirmed that prolonged QTD and QTcD rapidly decline after TAVI treatment, whereas SAVR slowly recovers QTD and QTcD. TAVI might therefore imply a higher postoperative arrhythmia risk than SAVR. However, in our cohort of $n=45$ patients undergoing TAVI, none experienced early postoperative ventricular arrhythmias.

\section{Comparison of TpTe Between TAVI and SAVR}

The peak of the $\mathrm{T}$ wave depicts the end of the epicardial action potential and the end of the $T$ wave shows the $\mathrm{M}$ cell action potential (midmyocardium). Hence, the action potential duration of the longest $\mathrm{M}$ cell determines the TpTe interval and serves as an index of TDR [11]. Similar to TpTe, the TpTe/QT ratio is a significant marker for evaluating the TDR under the conditions of long QT, short QT, Brugada syndrome, and acute ST-segment elevation [6]. In addition, TpTe, TpTe/QT, and TpTe/QTc exhibited a positive correlation with mean aortic gradient, and might be a predictor of AS severity [12]. Our findings indicate that TpTe, TpTe/QT, and TpTe/QTc were not prolonged in either the TAVI or SAVR groups, whereas QTD and QTcD were increased in the SAVR group, but not in the TAVI group. The contradiction between QTD, which shows the spatial distribution of repolarization, and TpTe, which shows the TDR, has been uncertain in the present study. Our hypotheses for this contradiction are as follows: (1) spatial inhomogeneity might be more susceptible to hypoperfusion (endocardial or subepicardial damage) compared to transmural inhomogeneity (a higher degree of myocardial damage), (2) the sample size of this study might be insufficient to detect the transmural inhomogeneity, (3) to detect the accurate transmural inhomogeneity, we might have to measure multiple TpTe or dispersion of TpTe [13]. To clarify the clinical significance of QTD and TpTe, further studies are needed.

\section{Limitations}

The long-term outcome of TAVI and SAVR remain controversial. In the short term, mortality and hospitalization are better in TAVI as compared to SAVR [1]. However, after 1 year, mortality worsened in the TAVI group as compared to SAVR [14]. To clearly determine the effect of TAVI on cardiac repolarization, longterm comparison between TAVI and SAVR is essential.

There was a significant difference in QT in lead V5 at 3 months. Such a statistical difference might arise from the reduction of participants in the late perioperative period of this observation. In our observation, the number of patients whose ECGs were obtained were gradually reduced, especially in SAVR (at 3 months: $n=15$, at 6 months: $n=14$ ) because of the transfer to another hospital. At 3 and 6 months, it was difficult to compare the ECG parameters in the present study. Further studies and 
accumulation of long-term outcomes are needed to determine the benefit of TAVI.

\section{CONCLUSIONS}

Our findings suggest that QTD decreased immediately after surgery in TAVI patients compared to SAVR patients. In terms of cardiac repolarization, TAVI might be a safer option as compared to SAVR, especially in the early postoperative period. However, further studies are needed to compare the long-term outcomes of TAVI and SAVR.

\section{ACKNOWLEDGEMENTS}

We would like to thank the participants of the study, and we would also like to thank Dr. Yasuo Haruyama, M.D., Ph.D. (Associate Professor, Department of Public Health, Dokkyo Medical University, Tochigi, Japan) for his assistance with the statistics.

Funding. No funding or sponsorship was received for this study or publication of this article. The Rapid Service Fee were funded by the authors.

Authorship. All named authors meet the International Committee of Medical Journal Editors (ICMJE) criteria for authorship for this article, take responsibility for the integrity of the work as a whole, and have given their approval for this version to be published.

Medical writing, Editorial, and other Assistance. Writing assistance in the preparation of this article was provided by Editage (http:// www.editage.jp). Financial support for this assistance was provided by the authors.

Disclosures. Satoru Chino, Eriko Yamanaka, Toshifumi Takasusuki, Shinsuke Hamaguchi, and Shigeki Yamaguchi have nothing to disclose.

Compliance with Ethics Guidelines. All procedures performed in studies involving human participants were in accordance with the ethical standards of the institutional and national research committee and the 1964 Helsinki Declaration and its later amendments or comparable ethical standards. Informed consent was obtained from all the participants and this study was approved by the ethics committee of Dokkyo Medical University (registration number: R-9-13). The study was registered at the UMIN Clinical Trials Registry (registration number: UMIN000033701).

Data Availability. The datasets generated during and/or analyzed during the current study are available from the corresponding author on reasonable request.

Open Access. This article is distributed under the terms of the Creative Commons Attribution-NonCommercial 4.0 International License (http://creativecommons.org/licenses/ by-nc/4.0/), which permits any noncommercial use, distribution, and reproduction in any medium, provided you give appropriate credit to the original author(s) and the source, provide a link to the Creative Commons license, and indicate if changes were made.

\section{REFERENCES}

1. Alsara O, Alsarah A, Laird-Fick H. Advanced age and the clinical outcomes of transcatheter aortic valve implantation. J Geriatr Cardiol. 2014;11:163-70.

2. Baumert M, Porta A, Vos MA, et al. QT interval variability in body surface ECG: measurement, physiological basis, and clinical value: position statement and consensus guidance endorsed by the European Heart Rhythm Association jointly with the ESC Working Group on Cardiac Cellular Electrophysiology. Europace. 2016;18:925-44.

3. de Bruyne MC, Hoes AW, Kors JA, Hofman A, van Bemmel JH, Grobbee DE. QTc dispersion predicts cardiac mortality in the elderly: the Rotterdam Study. Circulation. 1998;97:467-72.

4. Orlowska-Baranowska E, Baranowski R, Zakrzewski D, Kusmierczyk B, Rawczynska-Englert I. QT interval dispersion analysis in patients with aortic valve stenosis: a prospective study. J Heart Valve Dis. 2003;12:319-24. 
5. Gupta P, Patel C, Patel H, et al. T(p-e)/QT ratio as an index of arrhythmogenesis. J Electrocardiol. 2008;41:567-74.

6. Kors JA, Ritsema van Eck HJ, van Herpen G. The meaning of the Tp-Te interval and its diagnostic value. J Electrocardiol. 2008;41:575-80.

7. Kasapkara HA, Ayhan H, Durmaz T, et al. Shortterm effect of transcatheter aortic valve implantation on QT dispersion. Turk J Med Sci. 2015;45:615-8.

8. Kahraman S, Dogan A, Kalkan AK, et al. Evaluation of Tp-e interval, Tp-e/QT and Tp-e/QTc ratio in aortic valve stenosis before and after transcatheter aortic valve implantation. J Electrocardiol. 2018;51:949-54.

9. Sigmund AE, Fang Y, Chin M, et al. Postoperative tachycardia: clinically meaningful or benign consequence of orthopedic surgery? Mayo Clin Proc. 2017;92:98-105.

10. Nuis RJ, Turgut G, van der Boon RM, et al. Defective recovery of QT dispersion following transcatheter aortic valve implantation: frequency, predictors and prognosis. J Geriatr Cardiol. 2015;12:482-8.

11. Patel C, Burke JF, Patel H, Gupta P, Kowey PR, Antzelevitch C, Yan GX. Is there a significant transmural gradient in repolarization time in the intact heart? Cellular basis of the T wave: a century of controversy. Circ Arrhythm Electrophysiol. 2009;2:80-8.

12. Yayla Ç, Bilgin $M, A k b o \breve{g} a M K$, et al. Evaluation of Tp-E interval and Tp-E/QT ratio in patients with aortic stenosis. Ann Noninvasive Electrocardiol. 2016;21:287-93.

13. Xianpei W, Sha W, Chuanyu G, et al. Tpeak-Tend dispersion won't as a predictor for malignant arrhythmia events in patients with vasospastic angina. Int J Cardiol. 2017;249:61-5.

14. Armoiry X, Obadia JF, Pascal L, Polazzi S, Duclos A. Comparison of transcatheter versus surgical aortic valve implantation in high-risk patients: a nationwide study in France. J Thorac Cardiovasc Surg. 2018;156:1017-25. 